\begin{tabular}{ll}
\hline Bentham open & The Open Anesthesiology Journal \\
CrossMark & Content list available at: www.benthamopen.com/TOATJ/ \\
\hline
\end{tabular}

RESEARCH ARTICLE

\title{
The Confounding Effect of Assessor Ethnicity on Subjective Pain Reporting in Women
}

Jacob Miguel Vigil", Patrick Coulombe, Lauren Nikki Rowell, Chance Strenth, Eric Kruger, Joe Alcock, Kamilla Venner, Sarah See Stith and Joseph LaMendola

University of New Mexico, Mexico, USA

Received: August 26, 2016

Revised: November 01, 2016

Accepted: December 07, 2016

\begin{abstract}
The current study examines how subjective pain reporting is influenced by the concordant and discordant nature of the ethnic identities of pain expressers (participants) and pain assessors (experimenters). Three discomfort conditions that varied in stimuli intensity (Study 1: mild pain; Study 2: severe pain), and distraction components (Study 3) were used to assess whether pain intensity and tolerance reporting differ with the ethnic identification of the participant and the experimenter. Specifically, 87 Hispanic and 74 Non-Hispanic White (NHW) women $\left(18-51\right.$ yrs., $\left.M_{\text {age }}=20.0, S D=4.3\right)$ underwent a cold pressor pain task (CPT) after engaging in minimal procedural interactions with one of the 22 research experimenters (47\% Hispanic, $42 \%$ females). The procedural interactions with the experimenters included only consenting and instructions, with no interaction between experimenter and participant during the actual CPT. Random-effects models showed that between the $0 \%$ and $18 \%$ of the variance in pain sensitivity (intensity and tolerance scores) was attributable to characteristics of the experimenters. Controlling for self-esteem, baseline pain levels, and the gender of the experimenter, Hispanic subjects showed higher pain sensitivity (as marked by lower pain tolerance and higher pain intensity scores) following interactions with an NHW rather than a Hispanic experimenter in response to the most severe pain intensity stimuli. These results question the validity of common findings of ethnic differences in pain sensitivity from studies that have not accounted for the ethnic identity of the pain assessor (and the general communicative nature of pain reporting).
\end{abstract}

Keywords: Pain, Ethnicity, Social context, Pain measurement, Audience effects.

\section{INTRODUCTION}

Widespread findings in the basic and applied pain literatures that have attracted significant clinical attention and financial resources (e.g., in terms of federal funding aimed at reducing health disparities) are those of higher selfreported pain intensity among people with ethnic minority identities as compared to non-Hispanic Whites (NHWs) [1 3]. Current explanations of the ethnic differences in reported pain vary widely depending on the specialties and perspectives of the researchers at hand. Conventional models for explaining ethnic disparities in pain experiences focus on physiological causes (e.g., epigenetic profiles) [4, 5], as well as various and often inter-related psychological (e.g., social learning; coping strategies), sociological (e.g., health promotion behaviors, access to health services), and clinical factors including institutional-based models that highlight the role of under-treatment of pain in ethnic minority patients [6 - 10]. Another plausible yet overlooked explanation for the ethnic differences in pain sensitivity may be that they are actually the result of (often unavoidable) methodological confounds such as implicit social factors (ISFs) that influence how pain is commonly measured in scientific and medical research settings [11].

There are several converging lines of evidence in support of the hypothesis that the social context in which pain occurs affects the degree of pain that individuals perceive and report [11 - 13]. ISFs, such as characteristics of people in the immediate situational context, affect nearly every aspect of patient-provider and participant- experimenter

\footnotetext{
* Address correspondence to this author at the Logan Hall, 1 University of New Mexico Albuquerque, NM 87131-0001, USA; Tel: +505-277-0374; Fax: 505-277-1394; E-mail: vigilj@unm.edu
} 
interactions, including how people subjectively experience and report their pain, as well as how evaluators voluntarily (e.g., measure) and involuntarily respond (e.g., empathize) with the pain suffering of others [14]. Characteristics of experimenters, including gender and authority role, and even the passive and simulated presence of others (e.g., listening to a stranger's voice) can influence subjective pain ratings [15 - 21]. Unfortunately, the majority of previous research on pain sensitivity has been unable to control for these implicit ISFs, even in experiments that were otherwise operating under controlled laboratory conditions. It remains largely unknown how additional ISFs, especially those that play a foundational role in person perception, such as the ethnic identities of the persons evaluating pain in others, may influence pain reporting.

As described above, a common assumption is that racial / ethnic minority persons generally show higher pain sensitivity than NHWs; however, most of this research has been conducted comparing African-Americans to NHWs [3, 8, 22 - 24]. Less is known about how other ethnic groups, such as Latin-Americans / Hispanics, report their pain, and most of the previous studies on ethnic differences in pain sensitivity have not considered how characteristics of the experimenters themselves affect pain outcomes. Few studies have examined whether researcher ethnicity influences participants' pain reporting [25 - 27], and none of this work focused on Hispanics.

This is the first controlled study to examine how Hispanic and NHW women report cold pressor task (CPT) pain sensitivity after interacting with research experimenters with either a concordant or discordant ethnic identity. Three discomfort conditions that varied in stimuli intensity (Condition 1: mild pain; Condition 2: moderate pain), and distraction components (Condition 3) were designed to resemble expedient procedural interactions with intake healthcare/laboratory staff by limiting participant-experimenter interactions to only brief consenting and providing instructions; the experimental paradigm minimizes the potential confound of audience effects, because no researchers are present during the actual CPTs. Similar to previously reported findings [23, 24, 28], it was predicted that Hispanics would show greater pain sensitivity than NHWs with increasing pain intensity, yet that the pain reports would be influenced by the ethnicity of the experimenters who interacted with the participants prior to the actual CPT. It is possible, for example, that participants may respond to the ethnic identity of experimenters differently, depending on the participant's own ethnicity, which if observed, would confound the ability to reliably measure pain reports in clinical and experimental settings. This potential confound in pain reporting may be particularly pertinent to women due to their generally higher pain reports than men [14, 29], and findings that women may be more sensitive than men to contextual cues in research settings during standard discomfort tasks [20,30]. Other research shows that the influence of experimenter ethnicity may be different for females and males [27], therefore, the current study focused on the role of examiner ethnicity among women across three discrete experimental conditions.

\section{METHOD}

\section{Participants}

The University of New Mexico's Institutional Review Board approved protocols for each of the experiments and two forms of written consent were obtained from all participants. The first consent form described the general experimental protocol, and the second provided more detail on the CPT. Participants included a convenience sample of young women (U.S. citizens) who self-described being predominantly Hispanic/Latin-American or NHW. Participants with self-identified contraindication(s) to the CPT were excluded from the study. These contraindications included any illness related to a cardiovascular disorder (e.g., high blood pressure, heart disease, or dysrhythmia); history of fainting or seizures; history of frostbite; open cuts, sores or bone fractures on the limb to be immersed in water; or a history of Reynaud's phenomenon (excessively reduced blood flow in response to cold or emotional stress).

\section{Procedures}

Participants were assisted through the experimental protocol by 1 of the 22 research assistants, evenly split between self-described males and females (12 Hispanic, 10 NHW; $42 \%$ of the CPT Sessions were processed by a female experimenter and $47 \%$ by a Hispanic experimenter). Chi-square tests of independence showed that the ethnic identities and genders of the researchers did not differ for the NHW and Hispanic participants across the three experimental conditions $(p s>.27)$, except for the proportion of female researchers in the distraction condition $(p=.04)$ where a higher proportion of Hispanic participants were processed by male experimenters $(66 \%)$ than were processed by males among the NHW participants (39\%). 
The researchers followed a script for every phase of the experiment to minimize the possible influence of interpersonal factors and social skills associated with researcher characteristics (e.g., duration of conversations, eye contact, and warmth). Following the informed consent procedure, participants were escorted to an assessment room where they were left alone to complete a demographic questionnaire measuring gender, age, and ethnicity. Following the questionnaire, participants viewed a video that provided instructions (narrated by the first author) for performing the CPT without a researcher present. The video provided directions for using the cold pressor apparatus and indicated various pain ratings. Completing the survey and watching the instructional video took approximately 30 minutes.

Once participants finished watching the instructional video, they were led into the room with the cold pressor apparatus. The cold pressor room was fitted with a video camera for viewing the participants from a remote location, a cold pressor apparatus, and a laptop programmed with user-interfaced pain assessment software. The computer program was used to electronically measure participants' time latency and subjective pain intensity. Pain measurements were taken prior to engaging in the CPT (baseline pain intensity) and when they chose to discontinue the task due to an inability or unwillingness to tolerate the pain as described below (pain tolerance). Pain intensity ratings were also taken at thirty second intervals throughout the experiment. The participants were instructed to begin the task once the researcher left the CPT room. The participant then carried out the CPT without a researcher present, and the researcher observed the participant through a live video feed from the next room to ensure adherence to the cold pressor procedure. (The video feed was not explicitly revealed to participants.) This protocol enabled researchers to collect CPT data without being physically present during the CPT, which has been shown to influence experimental pain sensitivity [14, $16,18]$. None of the CPT sessions used in the present analyses were interrupted prematurely. Each of the participants spent a total of 5 to 7 minutes interacting with the experimenters (e.g., providing instructions, escorting the participants to various laboratory rooms) prior to the CPT and following the cold pressor task, participants were debriefed.

\section{Cold Pressor Task}

Cold pressor apparatus. Participants were seated in a chair between the pressor apparatus (left side) and the laptop computer (right side) in a small room $(2.0 \mathrm{~m} \times 2.5 \mathrm{~m})$. The mechanical CPT device was an Isotemp $6200 \mathrm{R} 28$ refrigerated bath circulator (reservoir size: $29 \mathrm{~cm} \times 16.5 \mathrm{~cm}$ x $22.4 \mathrm{~cm}$ ). The machine circulates the water automatically and maintains a consistent water temperature by dual heating and cooling actions. Because small differences in water temperature $\left(2^{\circ} \mathrm{C}\right)$ can have significant effects on pain sensitivity measures [31], all the participants in the current study experienced water temperatures within $0.5^{\circ} \mathrm{C}$ of each other.

Condition 1 (mild pain). The water temperature was set to $16^{\circ} \mathrm{C}$. Water at this temperature is temperate and should not cause pain expression under normal conditions (quasi- control condition).

Condition 2 (moderate pain). The water temperature was set to $5^{\circ} \mathrm{C}$ (known to produce a range of pain tolerance levels with only minimal ceiling effects) [32].

Condition 3 (moderate pain/orthotic distractor). As in Condition 2, the water temperature was set to $5^{\circ} \mathrm{C}$, but participants were required to insert a simple orthotic distractor device in their mouth prior to starting the CPT. The distractor device consisted of 4 (stacked) sterile, wooden tongue depressors (6" $\mathrm{x} .6875$ ") analogous to those used during standard medical checkups. Participants were asked to comfortably place two depressors on each side of the posterior mouth region (i.e., between the molars) pointed anteriorly. From this position, participants were instructed to "bite down in order to hold the depressors in place, so they are comfortable, yet firmly held" The participants were told if they felt the need to bite down harder on the orthotic device during the course of the experiment that they could do so. There was no direct reference to the CPT or the concept of pain more generally during these instructions, i.e., the participants were not told that biting down on the orthotic device would alleviate their pain or discomfort during the CPT.

Cold pressor procedures. As described in previous studies e.g., [21, 33] a pain assessment program was used and instructing participants to perform two simultaneous actions, when participants first begin the CPT (once the researcher has exited the room) and at the end of the task (indicating pain tolerance). Participants first indicated a baseline pain intensity score along a standard visual analog scale (VAS, 0-10 from no pain to worst pain imaginable), and simultaneously submerged their left hand into the CPT device. Participants record their pain tolerance by ending the task (lifting their hand out of the water) and clicking on a corresponding button on the computer screen. Participants also indicated their pain intensity in equal intervals (every $30 \mathrm{~s}$ ) throughout the CPT. 
Once the participants indicated their understanding of the CPT instructions, they were fitted with a finger pulsometer to monitor their heart rate during the CPT. Lastly, the researcher reminded the participant that they could begin the task after the researcher left the room; the researcher then left the cold pressor room and closed the door. The CPT was monitored via a video feed from another room, and the researcher re-entered the room once the participant terminated the CPT or after the maximum time limit of 5 minutes). Debriefing occurred after the participants heart rate had returned to resting and they no longer felt discomfort from the CPT.

\section{Data Analyses}

The pain scores included the participants' pain tolerance (measured in seconds post- submersion), and the pain intensity score sixty seconds into the CPT. Participants that completed the CPT between forty seconds and sixty seconds into the task and thus indicated a pain tolerance that did not last for a full minute were given an 8 out 10 for the subjective pain intensity score ( $16 \%$ of the sample), which was the most common maximum pain score reported by the sample. Higher intensity scores and lower tolerance scores are interpreted as indicating greater CPT pain sensitivity. Multilevel (random-effects) models were used to examine the separate effects of the ethnicity of the individual and experimenters, along with their interaction; participant's baseline (pre-manipulation) pain scores and the experimenters' gender were entered as covariates. The participants also completed a Rosenberg Self-Esteem Scale [34] to control for individual differences in trait self-esteem during the discomfort tasks, which are generally anxiety-provoking, and to better isolate the role of perceived ethnic identities (e.g., rather than social insecurities more generally) on pain reporting; the mean self-esteem score was included as a covariate. We used multilevel models in lieu of traditional linear regression/analysis of variance because observations are not independent and the sample size is limited. The same 22 experimenters were used for all 161 encounters, yet the goal is to generalize to any experimenter (and not only to the 22 experimenters used in this study). Participant and experimenter ethnicities were coded using an unweighted effects coding scheme $(\mathrm{NHW}=-0.5$, Hispanic $=+0.5)$, so that their effect in our analyses can be interpreted as main effects (averaging over the other ethnicity variable) [35]. The same coding scheme was adopted for the experimenter sex variable $($ Male $=-0.5$, Female $=+0.5$ ). Self-esteem was centered at the sample mean.

All of our analyses were conducted in R v3.2.2 [36], using restricted maximum likelihood estimation in the package lme4 v1.1-10 [37]. Significant interactions were decomposed using the multcomp package [38]. We also reported Cohen's $d$ (mean difference/mean standard deviation) [39] to provide an estimated effect size for the group comparisons without controlling for covariates and repeated measures among the experimenters.

In order to establish how much of the variation in the two pain scores (pain intensity and pain tolerance) is due to having interacted with a particular experimenter, we estimated null models for each of the pain experiments where each pain score is predicted only from a random intercept allowed to vary across experimenters. Only 3\% (mild pain condition), $2 \%$ (moderate pain condition), and $<1 \%$ (distraction condition) of the differences in pain intensity across participants were due to differences across experimenters. For pain tolerance, this figure was similar except in the moderate pain condition, where the proportion was much higher: $<1 \%$ (mild condition), 18\% (moderate condition), and $<1 \%$ (distraction condition) of the differences in pain tolerance were due to differences across experimenters. Overall, the individual-specific attributes of the experimenters do not appear to play as major a role in felt pain intensity. However, the individual-specific attributes of the experimenter with whom a participant interacts before experiencing moderate pain do appear to affect how long the participant is willing to endure the pain.

\section{RESULTS}

The final sample consisted of 161 women (54\% Hispanic; $18-51 \mathrm{yrs}$., $M_{\text {Hispanis }}=20.0, S D=4.4 ; M_{\text {мншs }}=19.9, S D=$ 4.2). A series of multilevel models were estimated, one for each of the two dependent variables (pain tolerance and pain intensity) across each of the three experiments using the participants' and experimenters' ethnic identity separately and the Participant Ethnicity x Experimenter Ethnicity interaction term as predictors (controlling for baseline pain score, experimenter gender, and participant self-esteem.) The results of these multilevel models are shown in Table 1. The first three columns of Table 1 show the results for pain tolerance. In the mild pain condition (Condition 1), there was no main effect of experimenter ethnicity or of participant ethnicity, but there was a significant interaction between the two ethnicity variables in predicting pain tolerance (a so-called "pure" interaction) [40]. A simple- slope analysis revealed that NHW participants tolerated pain for longer after interacting with an NHW experimenter rather than a Hispanic experimenter $(B=-95.0[S E=63.2], z=-1.50, p=.133, d=.88)$, whereas the pattern was reversed for Hispanic participants, who tolerated pain for longer after interacting with a Hispanic rather than NHW experimenter $(B=63.4$ 
$[S E=46.5], z=1.36, p=.173, d=-.66)$. However, neither effect taken individually reached statistical significance. Stronger effects emerged in the moderate-pain condition (Condition 2; see Table 1). In that condition, there was a main effect of experimenter ethnicity, averaging across NHW and Hispanic participants, participants who interacted with a Hispanic experimenter tolerated pain longer than participants who interacted with a NHW experimenter. However, this experimenter effect varied across NHW and Hispanic participants (again indicated by a significant interaction term). A simple-slope analysis revealed that the ethnicity of the experimenter had no effect on pain tolerance for NHW participants $(B=18.0[S E=34.4], z=0.52, p=.600, d=-.15)$, whereas Hispanic participants tolerated pain for longer after interacting with a Hispanic experimenter rather than an NHW experimenter $(B=152.5[S E=34.8], z=4.39, p<$ $.001, d=-.89$ ). Fig. (1) shows the pattern of this interaction.

Table 1. Results of the multilevel models predicting pain tolerance (first three columns) and pain intensity (last three columns) in the mild pain (Experiment 1), moderate pain (Experiment 2), and distraction (Experiment 3) conditions.

\begin{tabular}{|c|c|c|c|c|c|c|}
\hline \multirow[b]{2}{*}{ Predictor } & \multicolumn{3}{|c|}{ DV = Pain Tolerance } & \multicolumn{3}{|c|}{ DV = Pain Intensity } \\
\hline & Mild & Mod. & Dist. & Mild & Mod. & Dist. \\
\hline Intercept & $307.1 * * *$ & $124.7 * * *$ & $123.3 * * *$ & $3.6 * * *$ & $7.0^{* * *}$ & $6.3 * * *$ \\
\hline Experimenter Ethnicity & -15.8 & $85.2 * * *$ & 23.4 & 1.2 & -0.4 & -0.6 \\
\hline Participant Ethnicity & -51.3 & -33.5 & -24.2 & 1.0 & 0.0 & -0.1 \\
\hline Baseline Pain & -7.6 & $-27.6^{* *}$ & -4.9 & 0.0 & 0.3 & 0.2 \\
\hline Experimenter Sex & -1.5 & $-94.7 * * *$ & -17.7 & 0.3 & 0.7 & 0.3 \\
\hline Self-Esteem & -70.6 & $-56.3^{*}$ & 9.1 & 1.9 & 0.8 & -0.4 \\
\hline Exp. Ethnicity * Part. Ethnicity & $158.5^{*}$ & $134.4 * *$ & -109.5 & $-4.3^{*}$ & $-3.5^{* * *}$ & 1.5 \\
\hline Sample Size & 28 & 73 & 60 & 28 & 73 & 60 \\
\hline Complete Data & 28 & 71 & 59 & 28 & 53 & 42 \\
\hline
\end{tabular}

Note. Pain tolerance was measured in seconds and pain intensity was measured in self-reported pain level. Hypothesis tests are Wald $z$ tests. ${ }^{*} p<.05$; $* * p<.01 ; * * * p<.001$.

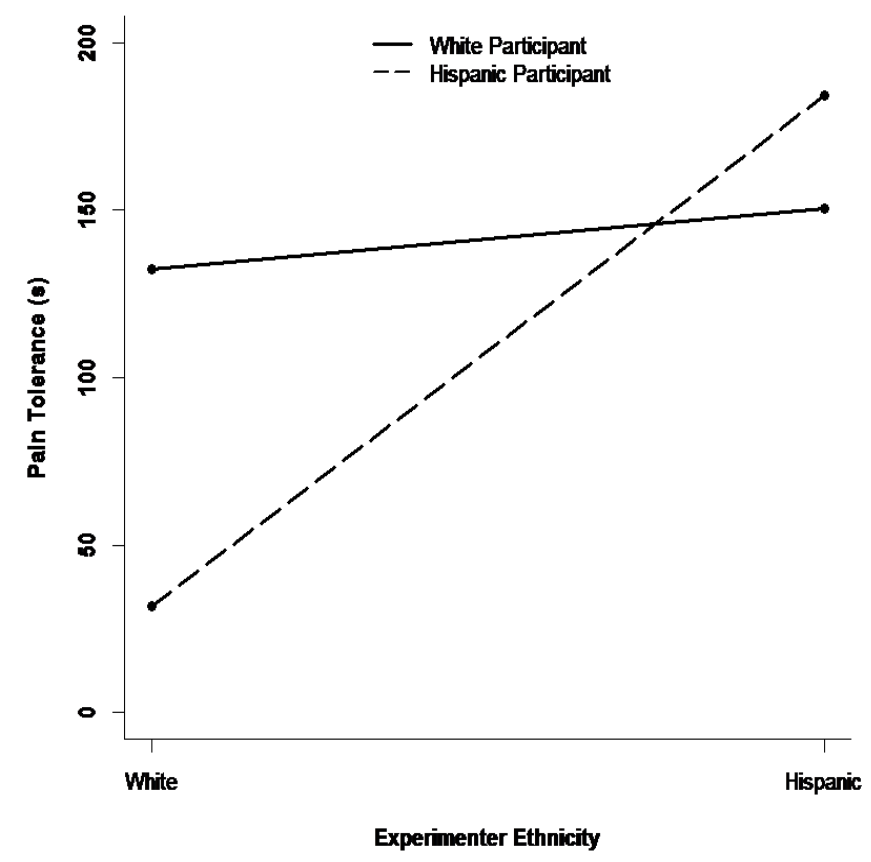

Fig. (1). Predicted pain tolerance (in seconds) in the moderate-pain condition as a function of experimenter ethnicity ( $x$ axis) and participant ethnicity (lines) when baseline pain score is 0 and self-esteem is average, averaging across experimenter genders.

There were other significant predictors of pain tolerance in the moderate-pain condition as well. Controlling for the other predictors in Table 1, participants who interacted with a female experimenter rather than a male experimenter tolerated pain for a shorter time. Also, baseline pain was negatively associated with pain tolerance, such that participants with higher levels of baseline pain withdrew from the pain task earlier. Finally, self-esteem was also associated with pain tolerance during the cold-pressor task, such that participants with lower self-esteem withdrew from 
the task earlier. In contrast, there were no significant predictors of pain tolerance in the distraction condition (Condition 3). The last three columns of Table 1 show the results for pain intensity. In the mild pain condition (Condition 1), as was the case for pain tolerance, there was no main effect of either experimenter or participant ethnicity, but a significant interaction emerged between the two ethnicity variables. In particular, a simple-slope analysis showed that NHW participants reported lower pain levels after interacting with an NHW experimenter rather than a Hispanic experimenter $(B=3.3[S E=1.6], z=2.07, p=.039, d=-1.45)$, whereas no such experimenter effect was observed among Hispanic participants $(B=-1.0[S E=1.2], z=-0.83, p=.405, d=.30)$. A similar result was obtained in the moderate pain condition (Condition 2). In that condition, there was again an interaction between experimenter and participant ethnicity in predicting pain intensity. A simple-slope analysis showed that NHW participants had a tendency to report lower pain levels after interacting with a NHW rather than Hispanic experimenter $(B=1.4[S E=0.8], z=1.69, p=.092, d=-.56)$, whereas the reverse pattern was observed among Hispanic participants, who reported experiencing lower pain intensity after interacting with a Hispanic rather than NHW experimenter $(B=-2.1[S E=0.8], z=-2.76, p=.006, d=1.05)$. Fig. (2) shows the pattern of this interaction. This is consistent with the pattern shown in Fig. (1), where Hispanic participants also displayed lower pain sensitivity (in the form of greater pain tolerance) after interacting with a Hispanic experimenter. Finally, there were no significant predictors of pain intensity in the distraction condition (Condition 3 ; see Table 1).

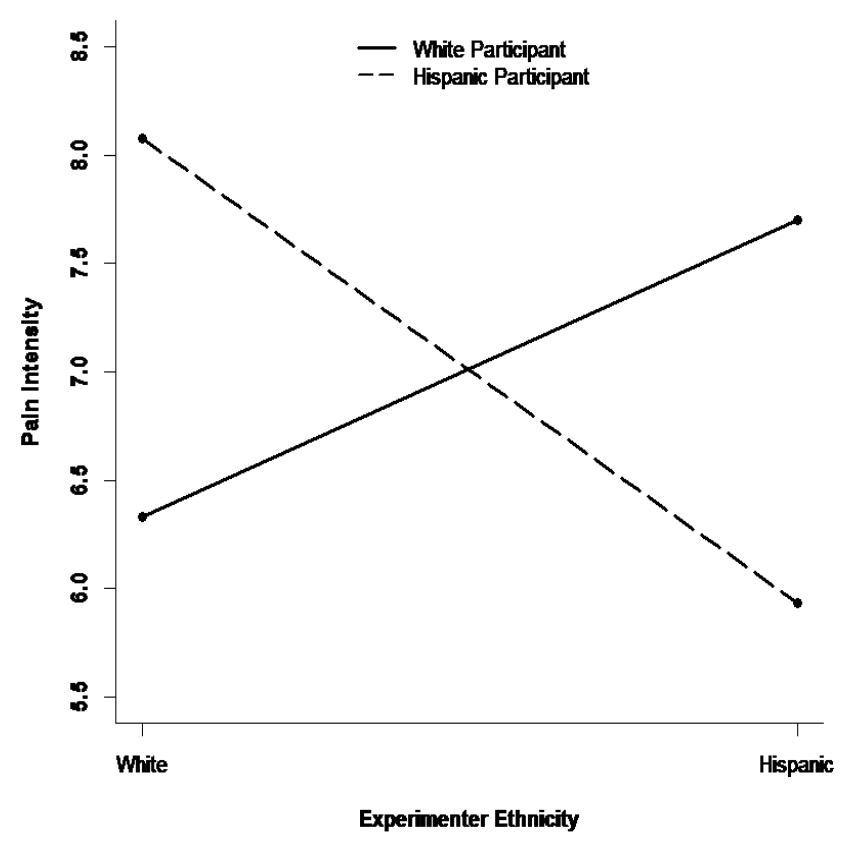

Fig. (2). Predicted pain intensity (self-reported pain level) in the moderate-pain condition as a function of experimenter ethnicity ( $x$ axis) and participant ethnicity (lines) when baseline pain score is 0 and self-esteem is average, averaging across experimenter genders.

\section{DISCUSSION}

This is the first study to show that the ethnic identity of laboratory experimenters who engage in minimal procedural interactions with research participants prior to an experimental pain task affects how people report their pain sensitivity. Specifically, we observed a general tendency of Hispanic participants to demonstrate lower pain tolerance and report higher pain intensity following interactions with a NHW rather than a Hispanic experimenter. This effect was not observed among NHW participants, was magnified in the moderate pain condition versus the mild pain condition, and appeared to be extinguished by a simple distraction technique. These unique results build on numerous previous investigations of ethnic differences in pain perception $[2,3]$ and stand in contrast to three previous investigations which did not find significant interactions between the ethnic identities of participants and experimenters (African American $v s$. NHW) and pain sensitivity [25 - 27]. This pain study was also among the first to focus on the influence of ethnicity during minimal procedural interactions with research experimenters, enabling us to account for the large proportion of variance in pain outcomes that was due to latent influences of experimenter characteristics. Nearly one-fifth of the variance in the pain tolerance scores was attributable to characteristics of the experimenters, demonstrating that multiple 
traits of experimenters who interact even minimally with research participants can affect experimental pain reporting under otherwise controlled laboratory conditions.

In the majority of experimental pain research studies, experimenter characteristics are not described in sufficient detail to gauge the likelihood of confounding effects, and experimenter effects are rarely the focus of investigation. Caution should therefore be applied when interpreting clinical pain assessments and experimental pain reporting when using paradigms that do not tightly control or account for interactions with different researchers and other ISFs. For example, because most previous research examining ethnic differences in pain reporting did not account for examiner characteristics, and given that the examiners in those studies were more than likely NHW, reevaluation of previous findings may be warranted. If anything, considerations of these results should be made when sampling patients for a clinical drug and other therapy trials, as a change in the experimental staff between initial appointments and follow-ups may result in misinterpreted changes in patient pain levels.

The current findings have several potential implications for health care providers. Pain is often considered the $5^{\text {th }}$ vital sign (joining temperature, heart rate, blood pressure and respiratory rate) and its measurement is considered central for effective and patient-centered care. Because many social cues likely influence how pain is perceived and reported, they likely obscure reliable pain measurements involving individuals and groups (e.g., patient prognosis, institutional outcomes). Variation alone in pain intensity and tolerance or even the reporting of pain intensity and tolerance could have treatment confounding consequences by causing illusory changes (improvement or a decline) in patient wellbeing, leading to unnecessary and potentially unhelpful fluctuations in care received by patients. Both the continued provision of care by doctors unaware of their own influences on patients' pain reports, as well as when health providers are on the side of caution, e.g., engage in "defensive care," can result in excessive care. These seemingly innocuous habits can be not only financially costly but also increase the risk of over-prescription of pain medication, prolong treatment periods, and increase insurance and coinsurance payments, possibly benefiting healthcare providers but certainly not benefiting patients or payers. Over-prescription of certain drugs such as opioid-based treatments (i.e., 'pain killers') can also carry unavoidable consequences such as medical and mental disturbances, risk of dependency, withdrawal, dysfunction and limitations in behavior, and risk of accidents (e.g., due to drowsiness) [41 - 46]. On the other hand, the results could also imply that alleviating pain for some patients might be achievable simply by changing the clinical personnel with whom that patient interacts. The possibility that the ethnicity of the experimenter or health care staff may affect Hispanics' pain ratings differently than NHWs is particularly important given that Hispanics are one of the largest growing ethnic groups in the United States [47] and thus highly representative of future patientprovider interactions.

While the underlying reasons for the influence of experimenter's ethnicity on subjective pain reporting remain speculative, they are broadly consistent with the psychological literature on social rejection, in-group/out- group perception, and basic social behavior strategies. Ethnic distribution falls along a social class gradient [48], and people may use some components of ethnicity as a heuristic to approximate in-group/out-group identities according to historical power roles in the local ecology [49]. Previous research suggests that individuals who describe themselves as having less societal influence attend (e.g., visually) to more hostile social threats in their environment [50, 51]. It is possible that a greater attention paid to contextual threats may very well manifest as a heightened awareness of pain, thus accounting for the decreased pain threshold and increased pain sensitivity of the minority participants when evaluated by NHW experimenters. Relatedly, due to the power imbalances between Hispanic and NHW in the U.S [48], Hispanics may show lower pain tolerance following interactions with NHW experimenters as an example of a reflexive and submissive signaling behavior, designed to avoid conflict with competitors when one is in a disadvantaged position $[12,13,52]$. The idea that behavioral adjustments are related to the demonstration of social dominance [48] is supported by overlapping neurobiological substrates of physical (experimental) pain and social pain reporting associated with interpersonal rejection and perceptions of social threat $[53,54]$.

The notion of stereotype threat [55] may be of particular importance when framing the results above. A stereotype threat occurs when cues in the environment highlight an individual's devalued group membership and results in a decrement in that individual's performance. The classic research example is racial / ethnic participants exhibiting poorer performance on an academic test when the instructions included implications of the test for measuring intelligence but not when instructions stated it would not reflect intelligence [55]. One possibility may therefore be that Hispanic participants experience stereotype threat or perceived inferiority biases following interactions with a NHW experimenter, thus resulting in 'poorer' pain performance, or ability to withstand the discomfort task (though the challenge of enduring the pain task was never made explicit). Another possibility is that the current participants had a 
tendency to perceive the NHW experimenters as more authoritarian than the Hispanic experimenters, which could have potentiated the impression that the experiment entailed greater (controlled) risk and intensity of noxious stimuli. Despite these uncertainties, the current findings are consistent with several converging lines of evidence that support the hypothesis that the social context in which pain occurs affects the degree of pain that individuals perceive and report [11 - 13]. Numerous other salient person perception characteristics (referred to here as 'ISFs') such as gender and physical stature likely also affect pain reporting in experimental and clinical settings [14, 27], with ethnicity being but one confounding factor.

Despite these potentially important implications, a discussion of the limitations of the study is warranted. A general limitation in the field is that most racial/ethnic studies are conducted with racial/ethnic groups in European and U.S. settings where NHW persons are the majority, so the current results may not generalize to countries where Latinos represent a majority rather than minority population. However, in the few studies that examine differences in ethnic populations where participants are tested in majority contexts (i.e., their home country), prototypical differences were minimal or even reversed [56, 57]. Therefore, although self-reported Hispanic ethnicity was used as a variable in this study, it is important to recognize that the Hispanic population is composed of many different cultural groups with diverse genetic make-ups [58 - 62], and the lack of measurement of acculturation [63] and other differences across Latino cultural groups (e.g., Mexican American, Cuban American, etc.) are limitations as well. It should be noted that the study was comprised of participants attending a State university with relatively similar cultural and ethnic backgrounds (i.e., with historical family origins from the Southwestern United States).

Finally, pain reactions caused by the CPT could be different than other types of pain sensations experienced in laboratory discomfort tasks and clinical settings, potentially limiting the generalizability of the observed effects of ethnicity. Methodologically, the study did not control for handedness, which has been shown to influence CPT measurements [64], as well as other individual-level characteristics and normative behaviors (e.g., exposure to cold water) of participants that may correlate with pain reporting. The sample was also comprised of healthy, young, female university students, whose responses may be different than other demographic groups. On the other hand, some benefits of using a relatively homogeneous sample is that it helped control for variability in pain reporting due to cultural background, financial status, comorbidities, and other potential confounding effects (e.g., participant gender).

Taking these potential limitations into account, the current findings challenge the belief that the concept of ethnicity exists in a vacuum, by showing it can have immediate or even latent influences on subjective pain reporting. Unless directly measured or controlled (e.g., via automated pain assessment procedures), examiner characteristics and other ISFs will likely remain confounding factors for the ability to reliably measure subjective pain reporting, provide costeffective and equitable patient care, and understand the underlying associations between pain and disease.

\section{CONFLICT OF INTEREST}

The authors confirm that this article content has no conflict of interest.

\section{ACKNOWLEDGEMENTS}

Declared none.

\section{REFERENCES}

[1] Betancourt JR, Green AR, Carrillo JE, Park ER. Cultural competence and health care disparities: key perspectives and trends. Health Aff (Millwood) 2005; 24(2): 499-505.

[http://dx.doi.org/10.1377/hlthaff.24.2.499] [PMID: 15757936]

[2] Green CR, Anderson KO, Baker TA, et al. The unequal burden of pain: confronting racial and ethnic disparities in pain. Pain Med 2003; 4(3): 277-94.

[http://dx.doi.org/10.1046/j.1526-4637.2003.03034.x] [PMID: 12974827]

[3] Rahim-Williams B, Riley JL III, Williams AK, Fillingim RB. A quantitative review of ethnic group differences in experimental pain response: do biology, psychology, and culture matter? Pain Med 2012; 13(4): 522-40. [http://dx.doi.org/10.1111/j.1526-4637.2012.01336.x] [PMID: 22390201]

[4] Hastie BA, Riley JL III, Kaplan L, et al. Ethnicity interacts with the OPRM1 gene in experimental pain sensitivity. Pain 2012 ; 153(8): 1610-9. [http://dx.doi.org/10.1016/j.pain.2012.03.022] [PMID: 22717102]

[5] Kim H, Neubert JK, San Miguel A, et al. Genetic influence on variability in human acute experimental pain sensitivity associated with gender, ethnicity and psychological temperament. Pain 2004; 109(3): 488-96. [http://dx.doi.org/10.1016/j.pain.2004.02.027] [PMID: 15157710] 
[6] Anderson KO, Green CR, Payne R. Racial and ethnic disparities in pain: causes and consequences of unequal care. J Pain 2009; 10(12): 1187-204.

[http://dx.doi.org/10.1016/j.jpain.2009.10.002] [PMID: 19944378]

[7] Burgess DJ, van Ryn M, Crowley-Matoka M, Malat J. Understanding the provider contribution to race/ethnicity disparities in pain treatment: insights from dual process models of stereotyping. Pain Med 2006; 7(2): 119-34. [http://dx.doi.org/10.1111/j.1526-4637.2006.00105.x] [PMID: 16634725]

[8] Campbell CM, Edwards RR. Ethnic differences in pain and pain management. Pain Manag 2012; 2(3): 219-30. [http://dx.doi.org/10.2217/pmt.12.7] [PMID: 23687518]

[9] Shavers VL, Bakos A, Sheppard VB. Race, ethnicity, and pain among the U.S. adult population. J Health Care Poor Underserved 2010; 21(1): $177-220$.

[http://dx.doi.org/10.1353/hpu.0.0255] [PMID: 20173263]

[10] Vigil JM, Alcock J, Coulombe P, et al. Ethnic disparities in emergency severity index scores among U.S. Veterans Affairs emergency department patients. PLoS One 2015; 10(5): e0126792. [http://dx.doi.org/10.1371/journal.pone.0126792] [PMID: 26024515]

[11] Vigil JM, Strenth C. No pain, no social gains: A social-signaling perspective of human pain behaviors. World J Anesthesiol $2014 ; 3(1)$ : 18-30. [http://dx.doi.org/10.5313/wja.v3.i1.18]

[12] Vigil JM. A socio-relational framework of sex differences in the expression of emotion. Behav Brain Sci 2009; 32(5): 375-90. [http://dx.doi.org/10.1017/S0140525X09991075] [PMID: 19825246]

[13] Vigil JM. The socio-relational framework of expressive behaviors as an integrative psychological paradigm. Behav Brain Sci 2009; 32: 408-28. [http://dx.doi.org/10.1017/S0140525X09990999]

[14] Vigil JM, Coulombe P. Biological sex and social setting affects pain intensity and observational coding of other peoples pain behaviors. Pain 2011; 152(9): 2125-30. [http://dx.doi.org/10.1016/j.pain.2011.05.019] [PMID: 21664763]

[15] Brown JL, Sheffield D, Leary MR, Robinson ME. Social support and experimental pain. Psychosom Med 2003; 65(2): 276-83. [http://dx.doi.org/10.1097/01.PSY.0000030388.62434.46] [PMID: 12651995]

[16] Kállai I, Barke A, Voss U. The effects of experimenter characteristics on pain reports in women and men. Pain 2004; 112(1-2): 142-7. [http://dx.doi.org/10.1016/j.pain.2004.08.008] [PMID: 15494194]

[17] Kleck RE, Vaughan RC, Cartwright-Smith J, Vaughan KB, Colby CZ, Lanzetta JT. Effects of being observed on expressive, subjective, and physiological responses to painful stimuli. J Pers Soc Psychol 1976; 34(6): 1211-8. [http://dx.doi.org/10.1037/0022-3514.34.6.1211] [PMID: 1003324]

[18] Levine FM, De Simone LL. The effects of experimenter gender on pain report in male and female subjects. Pain 1991; 44(1): 69-72. [http://dx.doi.org/10.1016/0304-3959(91)90149-R] [PMID: 2038491]

[19] McClelland LE, McCubbin JA. Social influence and pain response in women and men. J Behav Med 2008; $31(5)$ : $413-20$. [http://dx.doi.org/10.1007/s10865-008-9163-6] [PMID: 18587638]

[20] Vigil JM, Rowell LN, Alcock J, Maestes R. Laboratory personnel gender and cold pressor apparatus affect subjective pain reports. Pain Res Manag 2014; 19(1): e13-8. [http://dx.doi.org/10.1155/2014/213950] [PMID: 24367796]

[21] Vigil JM, Torres D, Wolff A, Hughes K. Exposure to virtual social stimuli modulates subjective pain reports. Pain Res Manag 2014; 19(4): e103-8.

[http://dx.doi.org/10.1155/2014/815056] [PMID: 24911175]

[22] Lu Q, Zeltzer L, Tsao J. Multiethnic differences in responses to laboratory pain stimuli among children. Health Psychol 2013; 32 (8): 905-14. [http://dx.doi.org/10.1037/a0032428] [PMID: 23668844]

[23] Rahim-Williams FB, Riley JL III, Herrera D, Campbell CM, Hastie BA, Fillingim RB. Ethnic identity predicts experimental pain sensitivity in African Americans and Hispanics. Pain 2007; 129(1-2): 177-84. [http://dx.doi.org/10.1016/j.pain.2006.12.016] [PMID: 17296267]

[24] Rowell LN, Mechlin B, Ji E, Addamo M, Girdler SS. Asians differ from non-Hispanic Whites in experimental pain sensitivity. Eur J Pain 2011; 15(7): 764-71. [http://dx.doi.org/10.1016/j.ejpain.2010.11.016] [PMID: 21561793]

[25] Edwards RR, Fillingim RB. Ethnic differences in thermal pain responses. Psychosom Med 1999; 61(3): $346-54$. [http://dx.doi.org/10.1097/00006842-199905000-00014] [PMID: 10367615]

[26] Hsieh AY, Tripp DA, Ji LJ. The influence of ethnic concordance and discordance on verbal reports and nonverbal behaviours of pain. Pain 2011; 152(9): 2016-22.

[http://dx.doi.org/10.1016/j.pain.2011.04.023] [PMID: 21616598]

[27] Weisse CS, Foster KK, Fisher EA. The influence of experimenter gender and race on pain reporting: does racial or gender concordance matter? Pain Med 2005; 6(1): 80-7. 
[http://dx.doi.org/10.1111/j.1526-4637.2005.05004.x] [PMID: 15669953]

[28] Mechlin MB, Maixner W, Light KC, Fisher JM, Girdler SS. African Americans show alterations in endogenous pain regulatory mechanisms and reduced pain tolerance to experimental pain procedures. Psychosom Med 2005; 67(6): 948-56. [http://dx.doi.org/10.1097/01.psy.0000188466.14546.68] [PMID: 16314600]

[29] Vigil JM. Current states of opinion and future directions on the epidemiology of sex differences in human pain. Pain Res Manag 2011; 16(5): 317-9. [http://dx.doi.org/10.1155/2011/280531] [PMID: 22059202]

[30] Vigil JM, DiDomenico J, Strenth C, et al. Experimenter effects on pain reporting in women vary across the menstrual cycle. Int J Endocrinol 2015; 2015: 520719. [http://dx.doi.org/10.1155/2015/520719] [PMID: 25892990]

[31] Mitchell LA, MacDonald RA, Brodie EE. Temperature and the cold pressor test. J Pain 2004; 5(4): $233-7$. [http://dx.doi.org/10.1016/j.jpain.2004.03.004] [PMID: 15162346]

[32] von Baeyer CL, Piira T, Chambers CT, Trapanotto M, Zeltzer LK. Guidelines for the cold pressor task as an experimental pain stimulus for use with children. J Pain 2005; 6(4): 218-27. [http://dx.doi.org/10.1016/j.jpain.2005.01.349] [PMID: 15820909]

[33] Vigil JM, Strenth C, Trujillo T, Gangestad SW. Fluctuating experimental pain sensitivities across the menstrual cycle are contingent on women's current romantic relationship status. PLoS One 2014; 9(3): e91993. [http://dx.doi.org/10.1371/journal.pone.0091993]

[34] Rosenberg M. Society and the adolescent self-image. Princeton, NJ: Princeton University Press 1965. [http://dx.doi.org/10.1515/9781400876136]

[35] Kraemer HC, Blasey CM. Centring in regression analyses: a strategy to prevent errors in statistical inference. Int J Methods Psychiatr Res 2004; 13(3): 141-51. [http://dx.doi.org/10.1002/mpr.170] [PMID: 15297898]

[36] R Development Core Team A language and environment for statistical computing; (Version 322) [Computer software] Vienna, Austria: R Foundation of Statistical Computing 2011 Available from: http://wwwR-projectorg/.

[37] Bates D, Maechler M, Bolker B, Walker S. Fitting linear mixed-effects models using lme4. J Stat Softw 2015; 67(1): 1-48. [http://dx.doi.org/10.18637/jss.v067.i01]

[38] Hothorn T, Bretz F, Westfall P. Simultaneous inference in general parametric models. Biom J 2008; 50(3): $346-63$. [http://dx.doi.org/10.1002/bimj.200810425] [PMID: 18481363]

[39] Cohen J. Statistical power analysis for the behavioral sciences. $2^{\text {nd }}$ ed. Hillsdale, NJ: Lawrence Erlbaum 1988.

[40] Keppel G. Wickens TD Design and analysis: A researcher's handbook. $4^{\text {th }}$ ed. Upper Saddle River, NJ: Prentice Hall 2004.

[41] Argoff CE, Viscusi ER. The use of opioid analgesics for chronic pain: minimizing the risk for harm. Am J Gastroenterol Suppl 2014; 2(1): 3-8. [http://dx.doi.org/10.1038/ajgsup.2014.3] [PMID: 25207607]

[42] Eisenberg E, McNicol ED, Carr DB. Efficacy and safety of opioid agonists in the treatment of neuropathic pain of nonmalignant origin: systematic review and meta-analysis of randomized controlled trials. JAMA 2005; 293(24): 3043-52. [http://dx.doi.org/10.1001/jama.293.24.3043] [PMID: 15972567]

[43] Hoppe JA, Kim H, Heard K. Association of emergency department opioid initiation with recurrent opioid use. Ann Emerg Med 2015; 65(5): 493-499.e4. [http://dx.doi.org/10.1016/j.annemergmed.2014.11.015] [PMID: 25534654]

[44] Langford RM. Pain management today - what have we learned? Clin Rheumatol 2006; 25(1)(Suppl. 1): S2-8. [http://dx.doi.org/10.1007/s10067-006-0311-5] [PMID: 16741780]

[45] Macintyre PE, Huxtable CA, Flint SL, Dobbin MD. Costs and consequences: a review of discharge opioid prescribing for ongoing management of acute pain. Anaesth Intensive Care 2014; 42(5): 558-74. [PMID: 25233168]

[46] Vowles KE, McEntee ML, Julnes PS, Frohe T, Ney JP, van der Goes DN. Rates of opioid misuse, abuse, and addiction in chronic pain: a systematic review and data synthesis. Pain 2015; 156(4): 569-76. [http://dx.doi.org/10.1097/01.j.pain.0000460357.01998.f1] [PMID: 25785523]

[47] Population Projections Table 4 Projections of the Population by Sex, Race, and Hispanic Origin for the United States: 2010 to 2050 U.S. Census Bureau: National Population Projections 2008 [Retrieved 2010-10-24].

[48] McLeod JD. Social stratification and inequality. In: Aneshensel CS, Phelan JC, Bierman A, Eds. Handbook of the sociology of mental health. New York: Springer 2013; pp. 229-53. [http://dx.doi.org/10.1007/978-94-007-4276-5_12]

[49] Park B, Rothbart M. Perception of out-group homogeneity and levels of social categorization: Memory for the subordinate attributes of ingroup and out-group members. J Pers Soc Psychol 1982; 42(6): 1051-68. [http://dx.doi.org/10.1037/0022-3514.42.6.1051] 
[50] Kraus MW, Horberg EJ, Goetz JL, Keltner D. Social class rank, threat vigilance, and hostile reactivity. Pers Soc Psychol Bull 2011; 37(10): 1376-88. [http://dx.doi.org/10.1177/0146167211410987] [PMID: 21653579]

[51] Vigil JM, Brophy S, Johnson T, Pendleton P, Rowell NL. Childhood correlates of protracted life dissatisfaction: Not all adversity hurts the same. In: Esposito A, Bianchi V, Eds. The Psychology of Happiness. Hauppauge, New York: Nova Science Publishers Inc 2013; pp. 111-23.

[52] Zuroff DC, Fournier MA, Moskowitz DS. Depression, perceived inferiority, and interpersonal behavior: evidence for the involuntary defeat strategy. J Soc Clin Psychol 2007; 26(7): 751-78. [http://dx.doi.org/10.1521/jscp.2007.26.7.751]

[53] Eisenberger NI. The pain of social disconnection: examining the shared neural underpinnings of physical and social pain. Nat Rev Neurosci 2012; 13(6): 421-34. [PMID: 22551663]

[54] Eisenberger NI, Jarcho JM, Lieberman MD, Naliboff BD. An experimental study of shared sensitivity to physical pain and social rejection. Pain 2006; 126(1-3): 132-8. [http://dx.doi.org/10.1016/j.pain.2006.06.024] [PMID: 16890354]

[55] Steele CM, Aronson J. Stereotype threat and the intellectual test performance of African Americans. J Pers Soc Psychol 1995; 69(5): 797-811. [http://dx.doi.org/10.1037/0022-3514.69.5.797] [PMID: 7473032]

[56] Nayak S, Shiflett S, Eshun S, Levine F. Culture and gender effects in pain beliefs and the prediction of pain tolerance. Cross-Cultural Res 2000; 34: 135-51. [http://dx.doi.org/10.1177/106939710003400203]

[57] Komiyama O, Kawara M, De Laat A. Ethnic differences regarding tactile and pain thresholds in the trigeminal region. J Pain 2007; 8(4): 363-9. [http://dx.doi.org/10.1016/j.jpain.2006.12.002] [PMID: 17275416]

[58] Hanis CL, Hewett-Emmett D, Bertin TK, Schull WJ. Origins of U.S. Hispanics. implications for diabetes. Diabetes Care 1991; 14(7): 618-27. [http://dx.doi.org/10.2337/diacare.14.7.618] [PMID: 1914811]

[59] Lewontin RC. The apportionment of human diversity. Evol Biol 1973; 6: 381-98.

[60] Tang H, Quertermous T, Rodriguez B, et al. Genetic structure, self-identified race/ethnicity, and confounding in case-control association studies. Am J Hum Genet 2005; 76(2): 268-75. [http://dx.doi.org/10.1086/427888] [PMID: 15625622]

[61] Weinick RM, Jacobs EA, Stone LC, Ortega AN, Burstin H. Hispanic healthcare disparities: challenging the myth of a monolithic Hispanic population. Med Care 2004; 42(4): 313-20. [http://dx.doi.org/10.1097/01.mlr.0000118705.27241.7c] [PMID: 15076807]

[62] Wilson JF, Weale ME, Smith AC, et al. Population genetic structure of variable drug response. Nat Genet 2001; 29(3): 265-9. [http://dx.doi.org/10.1038/ng761] [PMID: 11685208]

[63] Chan MY, Hamamura T, Janschewitz K. Ethnic differences in physical pain sensitivity: role of acculturation. Pain 2013; 154(1): 119-23. [http://dx.doi.org/10.1016/j.pain.2012.09.015] [PMID: 23149393]

[64] Pud D, Golan Y, Pesta R. Hand dominancy a feature affecting sensitivity to pain. Neurosci Lett 2009; 467(3): 237-40. [http://dx.doi.org/10.1016/j.neulet.2009.10.048] [PMID: 19853018]

(C) Vigil et al.; Licensee Bentham Open

This is an open access article licensed under the terms of the Creative Commons Attribution-Non-Commercial 4.0 International Public License (CC BY-NC 4.0)(https://creativecommons.org/licenses/by-nc/4.0/legalcode), which permits unrestricted, non-commercial use, distribution and reproduction in any medium, provided the work is properly cited. 